\title{
On kernels of cellular covers
}

\author{
Emmanuel D. Farjoun ${ }^{1}$, Rüdiger Göbel ${ }^{2}$, Yoav $\operatorname{Segev}^{1}$ and Saharon Shelah ${ }^{2,3}$
}

Dedicated to Avinoam Mann on the occasion of his retirement, 2006

\begin{abstract}
In the present paper we continue to examine cellular covers of groups, focusing on the cardinality and the structure of the kernel $K$ of the cellular map $G \rightarrow M$. We show that in general a torsion free reduced abelian group $M$ may have a proper class of non-isomorphic cellular covers. In other words, the cardinality of the kernels is unbounded. In the opposite direction we show that if the kernel of a cellular cover of any group $M$ has certain "freeness" properties, then its cardinality is bounded by $|M|$.
\end{abstract}

Mathematics Subject Classification (2000). 55P60.

Keywords. Cellular cover, infinite cardinal, free abelian group.

\section{Introduction and main results}

In this paper we continue the discussion of cellular covers in the category of groups begun in [FGS], [CFGS], where this notion is also motivated. Given a map of groups $c: G \rightarrow M$, we say that $(G, c)$ is a cellular cover of $M$ or that $c: G \rightarrow M$ is a cellular cover, if every group map $\varphi: G \rightarrow M$ factors uniquely through $c$, or, equivalently, the natural map $\operatorname{Hom}(G, G) \rightarrow \operatorname{Hom}(G, M)$, induced by $c$, is an isomorphism of sets. Explicitly this means that there exists a unique lift $\tilde{\varphi} \in \operatorname{End}(G)$ such that $\tilde{\varphi} \circ c=\varphi$ (maps are composed from left to right).

It has been shown before ([FGS], [FIR]) that cellular covers are values of general augmented $(F M \rightarrow M)$ and idempotent $(F \circ F=F)$ functors on the category of groups. More concretely, such functors are of the form cell $A_{A}(-)$, namely $A$-cellular approximation with respect to some group $A$.

\footnotetext{
${ }^{1}$ Partially supported by the Israel Science Foundation grant no. 712/07.

${ }^{2}$ Partially supported by project no. I-706-54.6/2001 of the German-Israeli Foundation for Scientific Research \& Development.

${ }^{3}$ This is the author's paper \# 892.
} 
The functors cell ${ }_{A}(-)$ had been used fruitfully in the category of groups, topological spaces and chain complexes over rings or DGAs (= differential graded algebras); compare, for example, Dwyer et al. [DGrI], [RSc], [FIR], Shoham (see [Sho]). The present results shade some light on the possible values of the functor cell $A_{A}(-)$ when $A$ is abelian. (We note that very different groups $A$ can give rise to the same functor.) It is possible that the values of all such functors (i.e. $\left\{\operatorname{cell}_{A} M \mid A\right.$ a group\}) on a fixed group $M$ yields only a set of results, up to isomorphism. In some topological analogous situations it has been shown that indeed only a set of values occurs (cf. [DP]). We have seen in [FGS], [CFGS] that this is the case when $M$ is a finite group, a finitely generated nilpotent group or a divisible abelian group. One aim of the present paper is to show that there are abelian groups $M$ for which $\left\{\operatorname{cell}_{A} M \mid A\right.$ an abelian group $\}$ is a proper class of isomorphism types.

Theorem 1. For any infinite cardinal $\lambda$, there exists an abelian group $M$ of cardinality $\lambda$ such that

(1) the isomorphism types of cellular covers $G \rightarrow M$ form a proper class. In fact,

(2) for every infinite cardinal $\kappa$ there is a surjective cellular cover $c_{\kappa}: G_{\kappa} \rightarrow M$ with $\left|\operatorname{ker} c_{\kappa}\right|=\kappa$. Furthermore,

(3) the group $M$ and the maps $c_{\kappa}$ can be chosen so that $\operatorname{Hom}\left(\operatorname{ker} c_{\kappa}, M\right)=0$ and $\operatorname{End}(M)=\mathbb{Z}$.

Theorem 1 is Theorem 2.10 of $\S 2$; its proof relies on Theorem 2.5 which may be of independent interest.

Let $c: G \rightarrow M$ be a cellular cover. In previous papers we have noticed that $G$ inherits several important properties from $M$ : First the kernel $K=\operatorname{ker} c$ is central in $G$, that is, $G$ is a central extension of $M$, and further, if $M$ is nilpotent, then $G$ is nilpotent of the same class; if $M$ is finite then so is $G$. In addition, we have classified all possible covers of divisible abelian groups ([CFGS]) and showed that when $M$ is abelian the kernel $K$ is reduced and torsion-free ([FGS, Theorem 4.7]). The case when $M$ is abelian was independently investigated in [BD] and [D]. Amongst other results it was shown there that when $M$ is (abelian and) reduced, $K$ is cotorsion free.

In [FGS] we have already observed that if $M$ is perfect, and $G$ is the so-called universal central extension of $M$ (so that $K$ is the Schur-multiplier), then $G \rightarrow M$ is a cellular cover, and, since any abelian group is a Schur-multiplier, in general, there is no restriction on $K$ (other than being in the center of $G$ and hence $K$ is abelian).

Note that the covers in Theorem 1 are very special covers in which the only map $\operatorname{ker} c_{\kappa} \rightarrow M$ from the kernel to $M$ is the zero map. This class of maps are both cellular covers and localization maps. Namely $c_{\kappa}: G_{\kappa} \rightarrow M$ is both a cellular cover and a localization. Recall that " $c$ is a localization" means that for any $\varphi \in \operatorname{Hom}(G, M)$ there is a unique corresponding $\tilde{\varphi} \in \operatorname{End}(M)$ such that $c \circ \tilde{\varphi}=\varphi$. Therefore, 
this class of localization-cellular maps $V \rightarrow W$ have the property that they induce isomorphisms on endomorphism sets: $\operatorname{End}(V) \cong \operatorname{Hom}(V, W) \cong \operatorname{End}(W)$.

The kernel $\operatorname{ker} c_{x}$ in Theorem 1 cannot be of arbitrary nature:

Theorem 2. For any cellular cover $c: G \rightarrow M$ (where $M$ is an arbitrary, not necessarily abelian, group), if the kernel $K$ of $c$ is a free abelian group then $|K| \leq|M|$.

In fact, the results in $\S 1$ (see Proposition 1.4) are somewhat more general than Theorem 2. We note that [FuG] continues the investigation of cellular covers of abelian groups begun in [CFGS] and in Theorem 1 of this paper, and in particular, further results on "large" cellular covers of "small" abelian groups are obtained there.

\section{Free kernels are small}

In this section we consider the kernel $K$ of a cellular cover $c: G \rightarrow M$. We impose some additional "freeness" assumptions on $K$. We show that under these restrictions the cardinality of $G$ is bounded in terms of the cardinality of $M$.

Definition 1.1 (compare with [EMe], p. 90). Let $K$ be an abelian group and $\alpha, \beta$ be cardinal numbers such that $\alpha \leq \beta$. We say that $K$ is weakly- $(\alpha, \beta)$-separable iff any subgroup $K_{1} \leq K$ of size $\leq \alpha$ is contained in a direct summand $K_{2} \leq K$ of size $\leq \beta$. Notice that when $\alpha=\beta$, then our notion coincides with the notion of (weakly) $\alpha^{+}$-separable group as in [EMe], p. 90. In this case we will say that $K$ is weakly- $\alpha$-separable (and not weakly $\alpha^{+}$-separable as in [EMe]).

We recall the following well-known fact.

Lemma 1.2. Let $K$ be a free abelian group. Then $K$ is weakly- $\alpha$-separable for every infinite cardinal number $\alpha$.

Proof. Let $K_{1}$ be a subgroup of $K$. Of course we may assume that $K_{1} \neq 0$. Let $\mathscr{B}$ be a basis of $K$ and for each $x \in K_{1}$ let $\mathscr{B}_{x} \subseteq \mathscr{B}$ be a finite subset such that $x \in\left\langle\mathscr{B}_{x}\right\rangle$. Let $K_{2}:=\left\langle\mathcal{B}_{x} \mid x \in K_{1}\right\rangle$. Then $K_{1} \leq K_{2},\left|K_{1}\right|=\left|K_{2}\right|$, and $K=K_{2} \oplus F$, where $F=\left\langle\mathcal{B} \backslash \bigcup_{x \in K_{1}} \mathscr{B}_{x}\right\rangle$.

Lemma 1.3. If $G, M$ are groups and $c \in \operatorname{Hom}(G, M)$ is surjective, then there exists $G_{1} \leq G$ such that $\left|G_{1}\right| \leq|M|+\aleph_{0}$ with $c\left(G_{1}\right)=M$.

Proof. For each $m \in M$ choose a preimage $g_{m} \in G$ (i.e. $c\left(g_{m}\right)=m$ ) and let $G_{1}=\left\langle g_{m} \mid m \in M\right\rangle$. 
Proposition 1.4. Let $c: G \rightarrow M$ be a cellular cover of the infinite group $M$ and set $K:=\operatorname{ker} c$. Let $\beta$ be a cardinal number such that $\beta \geq|M|$. Then

(1) if $K$ is weakly $(|M|, \beta)$-separable, then $|G| \leq \beta$; in particular,

(2) if $K$ is a free abelian group, then $|G| \leq|M|$.

Proof. Note that (2) is an immediate consequence of (1) and Lemma 1.2. It remains to prove (1). Notice that if we restrict the image and consider the map $c: G \rightarrow c(G)$ we still get a cellular cover. It follows that if $c(G)$ is finite, then $G$ is finite (see [FGS, Theorem 5.4]). We may thus assume without loss that $c$ is surjective. Let $G_{1} \leq G$ be a subgroup such that $c\left(G_{1}\right)=M$ and such that

$$
\left|G_{1}\right|=|M|,
$$

whose existence is guaranteed by Lemma 1.3 (note that since $M$ is infinite, $|M|+\boldsymbol{\aleph}_{0}=$ $|M|)$. Since $c\left(G_{1}\right)=M$, we have that

$$
G=K G_{1}
$$

Let $K_{1}:=G_{1} \cap K$; then $\left|K_{1}\right| \leq|M|$, so by hypothesis there exists a subgroup $K_{2} \leq K$ such that $K_{1} \leq K_{2},\left|K_{2}\right| \leq \beta$ and such that $K=K_{2} \times F$, for some $F \leq K$. It is easy to check that it follows that

$$
G=\left(G_{1} K_{2}\right) \times F .
$$

In particular, if $F \neq 1$, then, since $F \leq K$, $\operatorname{Hom}(G, K) \neq 0$, contradicting [FGS, Lemma 3.5 (c)]. Thus $F=1$, so $G=G_{1} K_{2}$ and hence $|G| \leq \beta$.

\section{Cellular covers with large kernels}

Before describing the main construction in Section C, we introduce some notation and definitions (Section A) and prove two existence result about "large" rigid abelian groups (Section B).

\section{A. Preliminaries}

Definition 2.1. Let $A$ be an abelian group, $q$ a prime and $\pi$ a set of primes. Then

(1) $A$ is $q$-reduced if $\bigcap_{i=1}^{\infty} q^{i} A=0$.

(2) $A$ is $\pi$-reduced if $A$ is $p$-reduced, for all $p \in \pi$.

(3) An element $a \in A$ is q-pure (in $A$ ) if $a$ is not divisible by $q$ in $A$.

(4) $A$ is $q$-divisible if each element $a \in A$ is divisible by $q$ in $A$. 
(5) An integer $n$ is a $\pi$-number, if $n$ is divisible only by primes from $\pi$ ( 1 and -1 are always $\pi$-numbers).

(6) A torsion element $a \in A$ is a $\pi$-element if the order of $a$ is a $\pi$-number (or $a=0)$.

(7) $A$ is a $\pi$-group, if each element of $A$ is a $\pi$-element.

(8) $\mathbb{Z}[1 / \pi]:=\mathbb{Z}[1 / p \mid p \in \pi]$ (and if $\pi=\emptyset$, then $\mathbb{Z}[1 / \pi]=\mathbb{Z}$ ).

Remark 2.2 (Tensor products, see [Fu]). (1) Let $A$ be a torsion free abelian group. Then $V:=\mathbb{Q} \otimes A$ is a vector space over $\mathbb{Q}$ which contains a copy of $A$ (cf. [Fu, Exercise 1(a), p. 260]). Thus we always think of $A$ as being contained in a vector space $V$ over $\mathbb{Q}$ such that $V / A$ is a torsion abelian group. Hence it makes sense to talk about the group $\left\langle A \cup\left\{\frac{a_{i}}{m_{i}} \mid i \in I\right\}\right\rangle$ where $I$ is an index set, $\left\{a_{i} \mid i \in I\right\} \subseteq A$ and $\left\{m_{i} \mid i \in I\right\} \subseteq \mathbb{Z} \backslash\{0\}$. This is the subgroup of $V$ generated by $A \cup\left\{\frac{a_{i}}{m_{i}} \mid i \in I\right\}$.

(2) Note that if $S \subseteq V$ and $\pi$ is a set of primes such that for each $s \in S$ there exists a $\pi$-number $n$ with $n s \in A$, then $\langle A \cup S\rangle / A$ is a $\pi$-group. In particular, for a subring $R \subseteq Q$ we view $R \otimes A$ as a subgroup of $V$ (cf. [Fu, Theorem 60.6, p. 260]) and if $R=\mathbb{Z}[1 / \pi]$, then $(R \otimes A) / A$ is a $\pi$-group.

(3) Note further that if $\pi_{1}$ and $\pi_{2}$ are disjoint sets of primes and $B \subseteq V$ is a subgroup containing $A$ such that $A$ is $\pi_{1}$-reduced and $B / A$ is a $\pi_{2}$-group, then $B$ is $\pi_{1}$-reduced.

Notation 2.3. Let $L$ be a torsion free abelian group and let $q$ be a prime. For $0 \neq x \in L$ we denote, using Remark $2.2(1)$,

$$
L \oplus_{x} \mathbb{Z}[1 / q]:=\left\langle L \cup\left\{\frac{x}{q^{i}} \mid 1 \leq i \in \mathbb{Z}\right\}\right\rangle .
$$

We write $H=x \mathbb{Z}[1 / q]$ for the subgroup of $L \oplus_{x} \mathbb{Z}[1 / q]$ consisting of the elements

$$
H:=\left\{\frac{m}{q^{i}} x \mid m \in \mathbb{Z} \text { and } 1 \leq i \in \mathbb{Z}\right\} .
$$

Remark 2.4. Assume $L$ is a torsion free abelian group, $q$ is a prime and $0 \neq x \in L$ is a $q$-pure element. Then

$$
L \oplus_{x} \mathbb{Z}[1 / q] \cong(L \oplus \mathbb{Z}[1 / q]) /\langle(-x, 1)\rangle .
$$

Furthermore, let $\widehat{M}$ be a group such that $\widehat{M}=L \oplus H$ where $L, H$ are subgroups of $\hat{M}, L$ is torsion free and $H$ is isomorphic to $\mathbb{Z}[1 / q]$ under an isomorphism taking some $0 \neq h \in H$ to 1 . Let $0 \neq y \in L$ be a $q$-pure element and let $M:=\widehat{M} /\langle y-h\rangle$. Then $M$ is isomorphic to the group $L \oplus_{y} \mathbb{Z}[1 / q]$ constructed in Notation 2.3. 
B. Existence of large rigid groups. The following is our main stepping stone for proving the existence of covers with arbitrarily large kernels.

Theorem 2.5. Let $P$ be a set of at least four primes, $Q$ its complementary set of primes and $\lambda$ any infinite cardinal. Then there is a torsion-free abelian group $H$ of cardinality $\lambda$ with the following three properties.

(1) $H$ is $Q$-reduced;

(2) if $Q_{0} \subseteq Q$ is a set of primes and $A$ is a torsion free abelian group containing $H$ such that $A / H$ is a $Q_{0}$-group, then $\operatorname{End}(A) \subseteq \mathbb{Z}\left[1 / Q_{0}\right]$;

(3) $H$ contains a free abelian group $F$ of rank $\lambda$ such that $H / F$ is a $P$-group.

Proof. Let $R:=\mathbb{Z}[1 / Q]$. By [GM] (see also [GT, Corollary 14.5.3(b), p. 577]), there exists an $R$-module $M$ of cardinality $\lambda$ such that $\operatorname{End}(M)=R$. Let $\mathcal{B}$ be a maximal $(\mathbb{Z})$-independent subset of $M$. We let

$$
F:=\langle\mathcal{B}\rangle \text { and } H:=\{x \in M \mid \text { there exists a } P \text {-number } n \in \mathbb{Z} \text { with } n x \in F\} \text {. }
$$

We claim that $H$ satisfies all the required properties. By construction (3) holds. Also, since $F$ is a free abelian group and since $H / F$ is a $P$-group, $H$ is $Q$-reduced (see Remark 2.2(3)), so (1) holds.

We now show (2). By construction, $M / H$ is a $Q$-group, so $R \otimes H=M$. Let $Q_{0}$ and $A$ be as in (2). Then $H \subseteq A \subseteq R \otimes A=R \otimes H=M$, and since $R \otimes A=M$, it follows that any endomorphism of $A$ extends to an endomorphism of $M$, thus $\operatorname{End}(A) \subseteq R$. Let $f \in \operatorname{End}(A)$ so that $f$ is multiplication by $\frac{m}{n}$, where $\operatorname{gcd}(m, n)=1$ and $n$ is a $Q$-number. Assume there exists a prime $q \in Q \backslash Q_{0}$ such that $q \mid n$. Then, after multiplying by an appropriate integer, we may assume that $n=q$. Writing $1=\alpha q+\beta m$, with $\alpha, \beta \in \mathbb{Z}$, we see that $\frac{1}{q}=\alpha+\beta \frac{m}{q}$, so multiplication by $\frac{1}{q}$ is an endomorphism of $A$. However, $q \notin Q_{0}, H$ is $Q$-reduced and $A / H$ is a $Q_{0}$-group, so Remark 2.2 (3) implies that $A$ is $q$-reduced. This is a contradiction. Thus $n$ is a $Q_{0}$-number, so $\operatorname{End}(A) \subseteq \mathbb{Z}\left[1 / Q_{0}\right]$ and (2) holds.

Remark. The set of primes $P$ in Theorem 2.5 is the set of primes that are used to construct the $\mathbb{Z}[1 / Q]$-module $M$ as in the beginning of the proof of the theorem. Thus we only work with the complimentary set of primes $Q$ when using the theorem to construct groups $L$ that have some desirable properties. Below we will fix the set $Q$ of primes which will be used for our constructions (in fact we only need 3 primes in $Q$, see Proposition 2.6 below). The set $P$ will be the complimentary set of primes.

In Section $\mathrm{C}$ below we use the following proposition whose proof relies on Theorem 2.5 . 
Proposition 2.6. Let $\lambda$ be any infinite cardinal and let $Q:=\left\{q_{K}, q_{L}, q\right\}$ be a set consisting of three primes. Then there exists an abelian group $L$ whose cardinality is $\lambda$ such that

(1) $L$ is $q_{K}$-reduced;

(2) $\operatorname{Hom}(L, K)=0$, for any abelian group $K$ which is $\left\{q_{L}, q\right\}$-reduced;

(3) there exists a q-pure element $y \in L$ such that the group $M:=L \oplus_{y} \mathbb{Z}[1 / q]$ satisfigs: is torsion free;

(b) $M$ is $q_{K}$-reduced;

(c) $\operatorname{End}(M)=\mathbb{Z}$.

Proof. We use Theorem 2.5 with $Q$ playing the role of $Q$ in that theorem. Let $R:=\mathbb{Z}[1 / Q]$ and let $H$ be as in Theorem 2.5. Let $F$ be as in part (3) of Theorem 2.5, and let $\mathscr{B} \subseteq F$ be a free generating set of $F$.

Pick $x \in \mathscr{B}$ and let $L_{1}:=\left\{\alpha_{x} x \in R \otimes H \mid \alpha_{x} \in \mathbb{Z}[1 /(P \cup\{q\})]\right.$,

$$
L_{2}:=\left\{v \in R \otimes H \mid v=\sum_{y \in \mathscr{B} \backslash\{x\}} \alpha_{y} y, \alpha_{y} \in \mathbb{Z}\left[1 /\left(P \cup\left\{q_{L}\right\}\right)\right]\right\} .
$$

and $L:=L_{1}+L_{2}$ (a direct sum). Notice that since $H / F$ is a $P$-group, $L \supseteq H$. Further, since $L / H$ is a $\left\{q_{L}, q\right\}$-group and since $H$ is $q_{K}$-reduced, Remark 2.2 (3) implies that $L$ is $q_{K}$-reduced so (1) holds.

Next, let $K$ be a $\left\{q_{L}, q\right\}$-reduced group and let $\eta \in \operatorname{Hom}(L, K)$. Then $\eta(\ell) \in$ $\bigcap_{i=1}^{\infty} q^{i} K$, for all $\ell \in L_{1}$, thus $\eta(\ell)=0$, since $K$ is $q$-reduced. Similarly, $\eta(\ell)=0$, for all $\ell \in L_{2}$, since $K$ is $q_{L}$-reduced, so (2) holds.

We now prove (3). We pick $y \in \mathscr{B} \backslash\{x\}$. Note that $y$ is $q$-pure in $L$. By construction $M$ is torsion free. Since $M / L$ is a $q$-group and since $L$ is $q_{K}$-reduced, Remark 2.2 (3) shows that $M$ is $q_{K}$-reduced.

To prove (3) (c), notice that every element in $M$ has the form

$$
\alpha_{x} x+\alpha_{y} y+\sum_{z \in \mathcal{B} \backslash\{x, y\}} \alpha_{z} z
$$

with

$$
\alpha_{x} \in \mathbb{Z}[1 /(P \cup\{q\})], \alpha_{y} \in \mathbb{Z}\left[1 /\left(P \cup\left\{q_{L}, q\right\}\right)\right], \alpha_{z} \in \mathbb{Z}\left[1 /\left(P \cup\left\{q_{L}\right\}\right)\right] .
$$

Note that $x$ is $q_{L}$-pure in $M$, while $z$ is $q$-pure in $M$, for all $z \in \mathscr{B} \backslash\{x, y\}$. Since $|\mathcal{B}|>2$, we see that $M$ is neither $q$-divisible nor $q_{L}$-divisible. Next, since $M / H$ is a $\left\{q, q_{L}\right\}$-group, Theorem $2.5(2)$ implies that $\operatorname{End}(M) \subseteq \mathbb{Z}\left[1 /\left\{q_{L}, q\right\}\right]$. Let $\varphi \in$ $\operatorname{End}(M)$, then there exists $\frac{m}{n} \in \mathbb{Q}$, with $\operatorname{gcd}(m, n)=1$ such that $n \geq 1$ is a $\left\{q_{L}, q\right\}$ number and such that $\varphi(x)=\frac{m}{n} x$, for all $x \in M$. Suppose $n \neq 1$ and let $p \in\left\{q_{L}, q\right\}$ such that $p \mid n$. Since $\frac{m}{n} x \in M$, for all $x \in M$ also $\frac{m}{p} x \in M$, for all $x \in M$ and then writing $1=\alpha m+\beta p, \alpha, \beta \in \mathbb{Z}$ we see that $\frac{1}{p} x=\frac{\alpha m}{p} x+\beta x \in M$. Thus $M$ is $p$-divisible. This contradicts an earlier remark. Hence $n=1$ and we see that $\operatorname{End}(M)=\mathbb{Z}$, so the proof of the proposition is complete. 
C. Constructing covers with arbitrarily large kernels. In this section we use Proposition 2.6 above to construct an abelian group $M$ and, for arbitrarily large cardinal $\kappa$, a cellular cover $G \rightarrow M$ whose kernel $K$ has cardinality $\kappa$. The group $M$ will be as in Proposition 2.6 (3).

We start with a very simple lemma that allows us to conclude that the canonical homomorphism $G \rightarrow G / K$ from the abelian group $G$ to the factor group $G / K$ is a cellular cover. The rest of the section is devoted to building arbitrarily large groups $K$ satisfying the conditions of the lemma (while $G / K$ remains fixed).

Lemma 2.7. Let $G$ be an abelian group and $K \leq G$ be a subgroup. Set $M:=G / K$ and let $c: G \rightarrow G / K$ be the canonical homomorphism. Assume that

(i) $\operatorname{End}(M) \cong \mathbb{Z}$;

(ii) $K$ is a fully invariant subgroup of $G$;

(iii) $\operatorname{Hom}(K, M)=0=\operatorname{Hom}(G, K)$.

Then $\operatorname{End}(G)=\mathbb{Z}$ and $c$ is a cellular cover.

Proof. Let $\mu \in \operatorname{End}(G)$. By (ii), $\mu(K) \leq K$ so $\mu$ induces $\hat{\mu} \in \operatorname{End}(M)$ defined by $\hat{\mu}(g+K)=\mu(g)+K$. By (i), there exists $n \in \mathbb{Z}$ such that $\hat{\mu}$ is multiplication by $n$. Thus the map $g \rightarrow(\mu(g)-n g)$ is in $\operatorname{Hom}(G, K)$, so by (iii) it is the zero map and it follows that $\mu$ is multiplication by $n$. This shows that $\operatorname{End}(G) \cong \mathbb{Z}$.

Let now $\varphi \in \operatorname{Hom}(G, M)$. Then by (iii), $\varphi(K)=0$, so $\varphi$ induces $\hat{\varphi} \in \operatorname{End}(M)$ defined by $\hat{\varphi}(g+K)=\varphi(g)$. Thus by (i) there is $n \in \mathbb{Z}$ such that $\varphi(g)=n g+K$, for all $g \in G$. Consequently, the map $\tilde{\varphi} \in \operatorname{End}(G)$ defined by $\tilde{\varphi}(g)=n g$ lifts $\varphi$, so any $\varphi \in \operatorname{Hom}(G, M)$ lifts. Since $\operatorname{Hom}(G, K)=0$, [FGS, Lemma 3.6] shows that $c$ is a cellular cover.

Lemma 2.8. Let $G$ be an abelian group containing subgroups $K$ and $\hat{M}$ such that $G=K+\hat{M}$. Set $M:=G / K$ and let $c: G \rightarrow M$ be the canonical homomorphism. Assume that

(i) $K$ is a torsion free fully invariant subgroup of $G$;

(ii) $K$ is an $R$-module for some subring $R \subset \mathbb{Q}$ and $\operatorname{End}(K)=R$;

(iii) $M$ is torsion free and $\operatorname{End}(M) \cong \mathbb{Z}$;

(iv) $\operatorname{Hom}(\widehat{M}, K)=0=\operatorname{Hom}(K, M)$;

(v) $K \cap \hat{M} \neq 0$.

Then $\operatorname{End}(G) \cong \mathbb{Z}$ and $c$ is a cellular cover.

Proof. We use Lemma 2.7. It only remains to show that $\operatorname{Hom}(G, K)=0$. Let $\mu \in \operatorname{Hom}(G, K)$. By hypothesis (iv), $\mu(\hat{M})=0$. By hypothesis (i), $\mu(K) \leq K$, so by hypothesis (ii) there exists $r \in R$ such that $\mu(v)=r v$, for all $v \in K$. Let $0 \neq v \in \hat{M} \cap K$. Then $r v=\mu(v)=0$, so since $K$ is torsion free, $r=0$, and it follows that $\mu(K)=0$ and then $\mu=0$. 
Proposition 2.9. Let $Q:=\left\{q_{K}, q_{L}, q\right\}$ be a set consisting of three primes. Let $K$ and $L$ be abelian groups and assume that

(i) $K$ is torsion free, it is $Q \backslash\left\{q_{K}\right\}$-reduced, and $\operatorname{End}(K)=\mathbb{Z}\left[1 / q_{K}\right]$.

(ii) L and the element $y \in L$ satisfy (1)-(3) of Proposition 2.6.

Let $0 \neq x \in K$ be an arbitrary element, and let

$$
G=(K \oplus L) \oplus(x-y) \mathbb{Z}[1 / q]
$$

be the group constructed in Notation 2.3, with $K \oplus L$ in place of $L$ and $x-y$ in place of $x$. Set

$$
H:=(x-y) \mathbb{Z}[1 / q] \text { and } \hat{M}=L+H .
$$

Then $G, K$ and $\hat{M}$ satisfy all the hypotheses of Lemma 2.8. In particular, the canonical homomorphism $c: G \rightarrow G / K$ is a cellular cover.

Proof. Clearly $G=K+\widehat{M}$. Now since $(K+L) \cap H=\langle x-y\rangle$, it is easy to check that

$$
K \cap \widehat{M}=\langle x\rangle .
$$

Note that $L \cap H=0$, because if $g:=n(x-y) \in L \cap H$, then $n(x-y) \in L$, which implies that $n x \in L$. But $K$ is torsion free and $K \cap L=0$, so $n=0$ and then $g=0$. Thus $\widehat{M}=L \oplus H$, also $x=y+(x-y)$ and $H \cong \mathbb{Z}[1 / q]$ by an isomorphism sending $(y-x) \rightarrow 1$, so by (I) and Remark $2.4, M \cong \hat{M} /\langle x\rangle \cong L \oplus_{y} \mathbb{Z}[1 / q]$. From hypothesis (ii) and Proposition 2.6 (3) it follows that

$$
M \text { is torsion free, } M \text { is } q_{K} \text {-reduced and } \operatorname{End}(M)=\mathbb{Z} .
$$

Since $K$ is $q_{K}$-divisible, we conclude that

$$
\operatorname{Hom}(K, M)=0,
$$

and also, since $M$ is $q_{K}$-reduced, we have $\bigcap_{i=0}^{\infty} q_{K}^{i} G=K$, so

$K$ is a fully invariant subgroup of $G$.

Next, since $K$ is $\left\{q_{L}, q\right\}$-reduced, $\operatorname{Hom}(L, K)=0$, by Proposition 2.6 (2). Similarly, since $H$ is $q$-divisible, $\operatorname{Hom}(H, K)=0$. Hence

$$
\operatorname{Hom}(\widehat{M}, K)=0 .
$$

Thus all hypotheses of Lemma 2.8 have been verified.

As a corollary to Proposition 2.9 we get Theorem 1 of the introduction. 
Theorem 2.10. Let $\lambda$ be any infinite cardinal. There exists an abelian group $M$ of cardinality $\lambda$ such that for any infinite cardinal $\kappa$ there exists a cellular cover $c: G \rightarrow M$ with $|\operatorname{ker} c|=\kappa$.

Proof. Let $Q:=\left\{q_{K}, q_{L}, q\right\}$ be a set consisting of 3 primes. Let $H$ be as in Theorem 2.5 with $|H|=\kappa$, and let $K:=\mathbb{Z}\left[1 / q_{K}\right] \otimes H$. Since $K / H$ is a $q_{K}$-group, and since $H$ is $Q \backslash\left\{q_{K}\right\}$-reduced, Remark $2.2(3)$ implies that $K$ is $Q \backslash\left\{q_{K}\right\}$-reduced. Further, by Theorem $2.5(2), \operatorname{End}(K)=\mathbb{Z}\left[1 / q_{K}\right]$. Thus $K$ satisfies hypothesis (i) of Proposition 2.9.

Proposition 2.6 guarantees the existence of a group $L$ of cardinality $\lambda$ as in hypothesis (ii) of Proposition 2.9. Let $G$ be as in Proposition 2.9 and set $M:=G / K$. By Proposition 2.9 the canonical map $c: G \rightarrow M$ is a cellular cover and since $\operatorname{ker} c=K,|\operatorname{ker} c|=\kappa$. Also $M \cong L \oplus_{y} \mathbb{Z}[1 / q]$, so $|M|=\lambda$ and the structure of $M$ is independent of the choice of $K$.

\section{References}

[BD] J. Buckner and M. Dugas, Co-local subgroups of abelian groups. In Abelian groups, rings, modules, and homological algebra, Lect. Notes Pure Appl. Math. 249, Chapman \& Hall/CRC, Boca Raton 2006, 29-37. Zbl 1108.20054 MR 2229098

[D] M. Dugas, Co-local subgroups of abelian groups. II. J. Pure Appl. Algebra 208 (2007), 117-126. Zbl 1108.20055 MR 2269833

[DGrI] W. G. Dwyer, J. P. C. Greenlees, and S. Iyengar, Duality in algebra and topology. Adv. Math. 200 (2006), 357-402. Zbl 05015044 MR 2200850

[DP] W. G. Dwyer and J. H. Palmieri, Ohkawa's theorem: there is a set of Bousfield classes. Proc. Amer. Math. Soc. 129 (2001), 881-886. Zbl 0963.55005 MR 1712921

[EMe] P. C. Eklof and A. H. Mekler, Almost free modules. North-Holland Math. Library 46, North-Holland, Amsterdam 1990. Zbl 0718.20027 MR 1055083

[FGS] E. D. Farjoun, R. Göbel, and Y. Segev, Cellular covers of groups. J. Pure Appl. Algebra 208 (2007), 61-76. Zbl 1105.55006 MR 2269828

[CFGS] W. Chachólski, E. D. Farjoun, R. Göbel and Y. Segev, Cellular covers of divisible abelian groups. Preprint, 2007.

[FIR] R. J. Flores, Nullification and cellularization of classifying spaces of finite groups. Trans. Amer. Math. Soc. 359 (2007), 1791-1816. Zbl 1112.55009 MR 2272149

[Fu] L. Fuchs, Infinite abelian groups, vol. I. Pure Appl. Math. 36, Academic Press, New York 1970. Zbl 0209.05503 MR 0255673

[FuG] L. Fuchs and R. Göbel, Cellular covers of abelian groups. Preprint 2006.

[GM] R. Göbel and W. May, Four submodules suffice for realizing algebras over commutative rings. J. Pure Appl. Algebra 65 (1990), 29-43. Zbl 0716.16015 MR 1065061 
[GT] R. Göbel and J. Trlifaj, Approximations and endomorphism algebras of modules. De Gruyter Exp. Math. 41, Walter de Gruyter, Berlin 2006. Zbl 05043166 MR 2251271

[RSc] J. L. Rodríguez and J. Scherer, Cellular approximations using Moore spaces. In Cohomological methods in homotopy theory (Bellaterra, 1998), Progr. Math. 196, Birkhäuser, Basel 2001, 357-374. Zbl 0987.55011 MR 1851263

[Sho] S. Shoham, Cellularizations over DGA with application to EM spectral sequence. Ph.D. thesis, The Hebrew University of Jerusalem, Jerusalem 2006.

Received November 23, 2006

E. D. Farjoun, Institute of Mathematics, The Hebrew University of Jerusalem, Givat Ram, Jerusalem 91904, Israel

E-mail: farjoun@math.huji.ac.il

R. Göbel, Fachbereich Mathematik, Universität Duisburg-Essen, 45117 Essen, Germany E-mail: ruediger.goebel@uni-due.de

Y. Segev, Department of Mathematics, Ben-Gurion University, Beer-Sheva 84105, Israel

E-mail: yoavs@math.bgu.ac.il

S. Shelah, Institute of Mathematics, The Hebrew University of Jerusalem, Givat Ram, Jerusalem 91904, Israel; and Department of Mathematics, Rutgers University, New Brunswick, NJ 08854-8019, U.S.A.

E-mail: shelah@math.huji.ac.il 